whilst Patient 4 underwent balloon dilatation and stenting, as the primary stenosis was discrete at the distal anastomosis site. After 6-months follow-up, Patient $3 \& 4$ recovered well with improved clinical status. Patient 5 had complete LV-PA conduit adhesion to the retro-sternum and demonstrated sub-systemic sub-pulmonary pressures by diagnostic catheterisation. It was agreed that the intervention risk outweighed any potential benefit and conservative management with follow-up would be most appropriate.

Discussion In our cases, despite knowing the exact anatomical location and conduit course, repeat echocardiography remained non-diagnostic. This surgical approach was first described in 1976, and there are case reports on only 15 patients, whereby, direct LV-PA resection was not felt to be surgically feasible due to abnormal mitral valve chordal attachments and/or long fibromuscular tunnel-type obstruction. It remains technically challenging to directly relieve native LV-PA obstruction without having detrimental impact on systemic right ventricular function, tricuspid valvular competence and electro-physiologically. But as ACHD practice has evolved, it is now actually an anatomical problem which doesn't necessarily always need a 'surgical-fix'.

Conclusion Awareness of this historical surgical approach to managing native LV-PA obstruction is important to understanding physiology and the potential long-term sequelae in these patients. They present a particular imaging challenge, especially via echocardiography, but an important finding to be comprehensively assessed through multimodal cross-sectional imaging. Although the technique is gone, it shouldn't be forgotten. Conflict of Interest None

\section{SEVERE AORTIC STENOSIS MANAGEMENT IN A TERTIARY CARDIAC CENTRE}

Norildin Al-Refaie, Waqas Jarral, Abhishek Shetye, Mark Cassar, Jim Newton. Oxford University Hospitals NHS Trust, Oxford, UK

\subsection{6/heartjnl-2021-BCS.30}

Introduction Aortic stenosis (AS) is the most common valvular heart disease in developed countries with an estimated prevalence of $3 \%$. According to ESC/EACTS guidelines, invasive management is recommended in patients with severe symptomatic AS, because of dismal spontaneous prognosis. It was reported that the average survival durations of patients with severe AS after developing symptoms such as angina, syncope, and shortness of breath were only 5, 3 and 2 years respectively if managed conservatively. Management of asymptomatic severe AS remains controversial.

Purpose Assess the prevalence of symptoms in patients with severe AS, and their management plans in relation with symptoms and age.

Method Retrospective data analysis of 259 patients with severe AS in a tertiary cardiac centre. Clinic notes, operation sheets, procedure reports and discharge summaries were the main source of data.

Results 199 (76.8\%) patients were symptomatic (shortness of breath, chest pain, dizziness or syncope). 143 (71.8\%) of these underwent intervention (surgical aortic valve replacement (SAVR), transcatheter valve implantation (TAVI) or Valvuloplasty). 56 (28.2\%) were managed conservatively (reasons for this being high risk for intervention, technical inadequacy for TAVI and patient informed decision). 9 (4.5\%) patients passed away during the study duration (all were managed conservatively).60 (23.2\%) patients were asymptomatic, of which 8 $(13.3 \%)$ underwent intervention. None of medically managed asymptomatic patients passed away during the study period. Average age for symptomatic patients who had surgical AVR

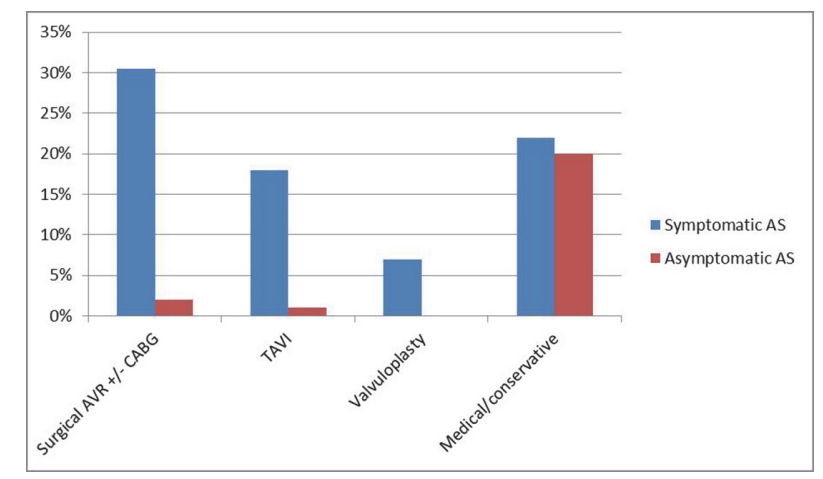

Abstract 30 Figure 1

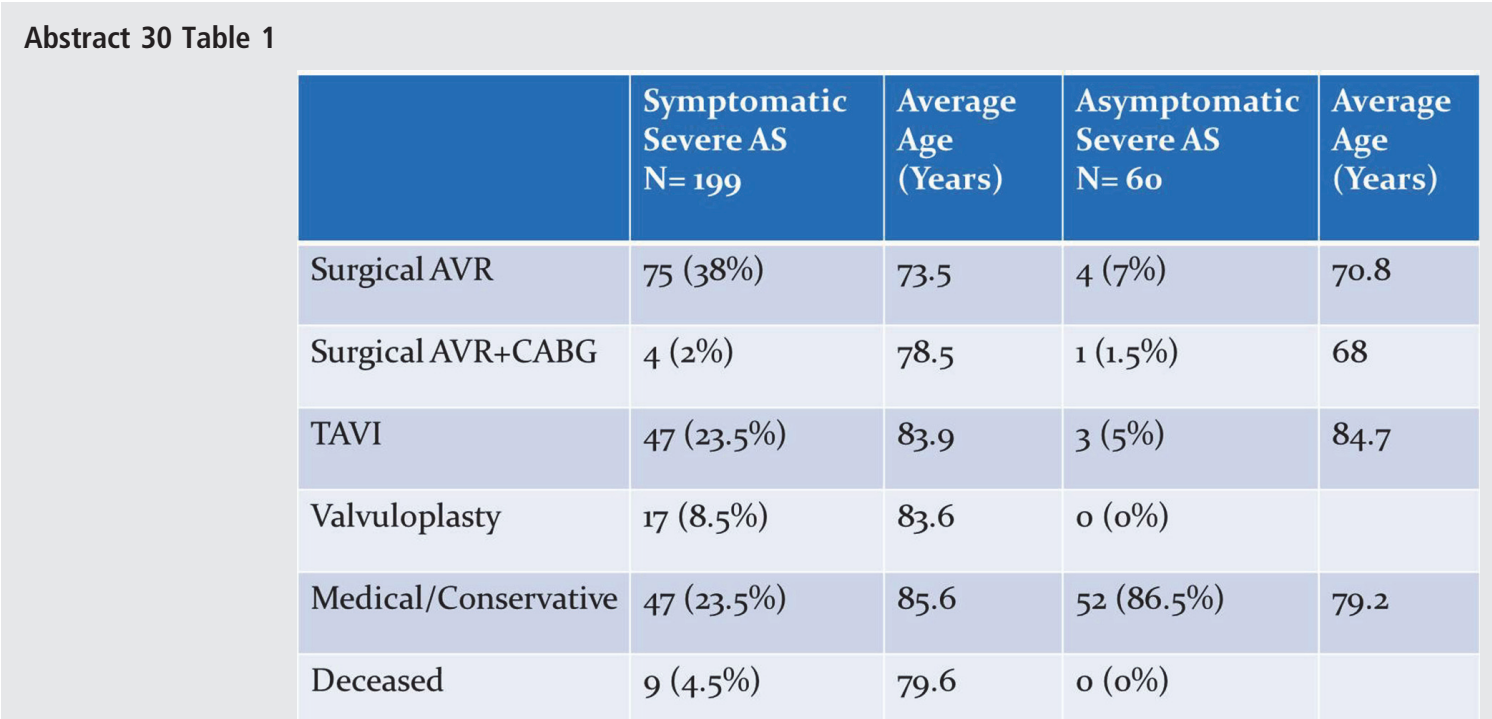


$\pm \mathrm{CABG}$ was 73.8 years, whilst average age for patients who had TAVI was 83.9 years, otherwise average age for valvuloplasty and medical treatment groups were 83.6 and 85.6 years respectively.

Conclusion Almost 3 out of each 4 patients with severe AS followed up in a tertiary cardiac centre were symptomatic, and more than two thirds of them underwent intervention. On average patients who had surgical AVR \pm CABG were almost a decade younger than patients in other treatment groups including patients who had TAVI, valvuloplasty and medical treatment. Most of the patients with asymptomatic severe AS were managed conservatively. This study highlights the high prevalence of symptoms within this population, and has proven useful to estimate the number of annual procedures required based on the number of symptomatic patients. Conflict of Interest None

\section{COMMUNITY-ACQUIRED CULTURE-NEGATIVE ENDOCARDITIS: CLINICAL CHARACTERISTICS AND OUTCOMES}

Anass Maaroufi, Hatim Zahidi, Marwa Abdulhakeem, Rachida Habbal. Ibn Rochd University Hospital, Casablanca, Morocco

10.1136/heartjnl-2021-BCS.31

Introduction Community-acquired culture-negative endocarditis is a rare entity poorly studied.

Objectives We studied the clinical characteristics, in-hospital mortality, and short-term prognosis of patients with culturenegative endocarditis.

Methods In total, 104 episodes of definite endocarditis according to duke criteria, were studied (2014-2019). We compared the clinical, laboratory, and echocardiography characteristics and the complications and survival rates of patients with culture-negative and culture-positive endocarditis.

Results Culture-negative endocarditis occurred in 76/104 (73\%) episodes. Compared with the culture-positive endocarditis patients, the time elapsed between first symptoms and admission was lower in patients with culture-negative endocarditis, a mean of 20 days vs 30 days in culture positive group $(\mathrm{p}<0.05)$, and these patients also had lower C-reactive protein levels at admission (99 vs $120 \mathrm{mg} / \mathrm{dl}$ ) ( $<<0.05$ ). in hospital stay was not diffirent between the two groups with a mean of days of hospitalization of 27 days . However in-hospital mortality rates were higher in culture-negative versus culture-positive patients ( $15 \%$ vs $11 \%$ mortality rate) . as for comlication rates Severe sepsis and vascular comlication (stroke , splenic infarction) were higher in the negative culture group with $7.9 \%$ vs $3.1 \%$ in the positive culture groupe $\mathrm{p}<0.05$.

Conclusions Culture-negative endocarditis patients presented with lower levels of C-reactive protein at admission and required less time for hospital admission, however presented a higher rate of in-hospital mortality and complications compared to culture-positive endocarditis patients.

Conflict of Interest none

\section{INFECTIVE ENDOCARDITIS: A REVIEW OF THE PATIENT JOURNEY AT A DISTRICT GENERAL HOSPITAL (DGH)}

Leanne Eveson, Heather Nicholson, Amani Patel, Catherine MacFarlane, Ann Parker, David Garner, Lydia Sturridge. Frimley Park Hospital, Frimley, UK
Introduction Infective endocarditis (IE) is a rare and potentially fatal infection. Patients often present with generalised symptoms resulting in delays in diagnosis. Complications are common and patients can have long inpatient stays due to intravenous antibiotic requirement. The POET study highlighted non inferiority of oral antibiotic switch in stable patients with left sided IE. The aim of our study is to characterise the IE patient population at our $\mathrm{DGH}$, analyse their management, review adherence of our Endocarditis Team to the ESC 2015 guidelines (figure 1) and to review discharge information and advice, to identify areas for improvement.

Methods This was a retrospective study of medical records for patients treated at our DGH with a diagnosis of IE between 1 Oct 2019 and 30 Sep 2020. Demographics, patient characteristics (table 1), IE risk factors, presentation, management, discharge information, patient feedback, and cost analysis was carried out. Adherence of our Endocarditis Team to ESC 2015 guidelines was reviewed.

Results Between October 2019 and September 2020, 14 patients were diagnosed with IE according to the Duke Criteria. The median age was $75(60-89)$ years and $68.7 \%$ of patients were male. 7 patients had a prosthetic valve and 3 had a cardiac device in situ. All patients had CVR risk factors, $50 \%$ had T2DM, 36\% had renal disease. Only 2 patients had documentation of dental history. The majority of patients $(n=12)$ presented via the Emergency Department. $86 \%$ of patients completed a 6 -week antibiotic course. Only $14 \%$ of patients were managed as outpatients. $21 \%$ of patients required surgical management. All patients were reviewed by the Endocarditis Team. All patients were followed up appropriately. Only 1 patient had documented advice regarding dental care. 1 patient died and many patients had complications (figure 2). IE patient support group feedback highlighted concerns regarding delay in diagnosis, challenges of a long inpatient stay and benefits of hearing from other patients about their experiences.

\section{Abstract 32 Table 1 Patient characteristics}

Peripheral stigmata of IE

Echocardiographic evidence of vegetation

Role of the 'Endocarditis Team'

I. The 'Endocarditis Team' should have meetings on a regular basis in order to discuss cases, take surgical decisions, and define the type of follow-up.

2. The 'Endocarditis Team' chooses the type, duration, and mode of follow up of antibiotic therapy, according to a standardized protocol, following the current guidelines.

3. The 'Endocarditis Team' should participate in national or international registries, publicly report the mortality and morbidity of their centre, and be involved in a quality improvement programme, as well as in a patient education programme.

4. The follow-up should be organized on an outpatient visit basis at a frequency depending on the patient's clinical status (ideally at I, 3, 6 , and 12 months after hospital discharge, since the majority of events occur during this period ${ }^{57}$ ).

Abstract 32 Figure 1 